\title{
Inhibition of Biofilm Formation in Some Pathogenic Gram Negative Bacteria by Anti-quorum Sensing Compounds
}

\author{
Fatma, A. A. Shaban, Zeinab. M. H. Kheiralla, Abeer, A. Rushdy, Khaled, Z. El- \\ Baghdady and
}

Botany department, Faculty of women for Arts, Science and Education, Ain Shams University.

\begin{abstract}
:
The present study included collecting of 125 clinical bacterial isolates from different sources such as pus, sputum, nasal polyps, urine and blood. The collected bacterial isolates were preliminary identified by biochemical tests as 44 Pseudomonas spp., 35 Klebsiella spp., 18 E. coli, 17 Acinetobacter spp., 8 Proteus spp. and 3 Enterobacter spp., The test for biofilm formation in these isolates was carried out by using three different methods that finally resulted in 27 positive biofilm forming isolates. Production of quorum sensing signal molecules (acyl homoserine lactone) was detected in 20 isolates using gas chromatography mass spectrum (GC/MS); These isolates were 3 Pseudomonas spp., 6 Klebsiella spp., 8 E. coli and 3 Enterobacter spp., The anti-biofilm activity of some plant extracts was carried out using tube method. It showed that Syzygium aromaticum was the most effective one and inhibited biofilm formation in all isolates followed by Allium sativum. Syzygium aromaticum extract also inhibited quorum sensing production in the selected 20 bacterial isolates. From the above results, Syzygium aromaticum extract was recommended to be used as an active antibacterial agent against multidrug resistant bacteria due to its ability to inhibit biofilm formation and quorum sensing signal production. Finally, the most potent bacterial isolates were genetically identified by $16 \mathrm{~S}$ rRNA gene sequencing.
\end{abstract}

Keywords: Quorum sensing, Acyl homoserine lactone, Gram Negative bacteria \& Biofilm.

\section{Introduction}

Biofilm is an exopolysaccharide, a slime matrix around multiple layers of cells. Biofilm formation is considered to be two-step process in which the bacteria first adhere to a surface by production of exopolysaccharides, followed by multiplication to form multilayered biofilm $[1,2]$. Within a biofilm, bacteria communicate with each other by production of chemotactic particles or pheromones, a phenomenon called quorum sensing [3]. Microorganisms growing in a biofilm are intrinsically more resistant to antimicrobial agents than planktonic cells. High antimicrobial concentrations are required to inactivate organisms growing in a biofilm, as antibiotic resistance can increase 1,000 fold. [4]. Quorum sensing (QS) is a complex environmental sensing system employed by bacteria to communicate among themselves and thereby regulate their population activities in response to various stimuli. The QS mechanism depends on the synthesis and release of chemical signals into the environment and on the detection of these signals as a function of cell population density.

*Corresponding author: fatma.abdeltawab@women.asu.edu.eg 


\section{Materials and Methods}

Such group behavior results in altered gene expression that drives the activities of the bacteria in a coordinated manner $[5,6]$. Bacteria synthesize chemical signals that include a wide variety of small molecules [7]. Of these, the N-acylhomoserine lactones (AHLs) are the most commonly used by Gram-negative bacteria for bacterial communication. The biosynthesis and effects of AHLs depend primarily on the activity of the LuxI and LuxR protein families, respectively. After AHLs are produced by LuxI enzymes (AHL synthases), they diffuse across bacterial membranes and accumulate externally until reaching high local concentrations. At a given threshold intracellular concentration, the AHL binds to a LuxR response regulator forming a complex that regulates gene expression [8,9] such as biofilm formation. Therefor targeting inhibition of quorum sensing resulted in inhibition of biofilm formation and stop pathogenicity and resistance of pathogenic gram negative bacteria against the common used antibiotics.

The aim of this work was to inhibit biofilm formation via targeting quorum sensing system in some pathogenic Gram negative bacteria by using some medicinal plants.

\subsection{Bacterial isolates}

A total number of 125 clinical isolates were collected from microbiology laboratory in El-Demerdash hospital. All of these isolates were Gram negative bacteria.

\subsection{Plant materials}

Twelve plant species were exploited in this study some of them were collected from the garden of Botany Department, Faculty of Science, Ain Shams University and the others were purchased from local commercial markets in Cairo city, Egypt.

\subsection{Preliminary Identification of clinical isolates}

Preliminary screening was carried out according to Bergey's Manual of Determinative Bacteriology [10].

\subsection{Detection of biofilm formation}

Biofilm formation was detected by three different methods; Congo Red Agar method according to [11], Micro-titer plate assay [12] and tube method [13].

\subsection{Inhibition of biofilm formation}

The antibacterial activity of some plant extracts was detected using the standard well agar diffusion method [14] and also tested for their ability to inhibit biofilm formation in the selected bacterial isolates using tube method [13].

\subsection{Antibiotic susceptibility test}

The selected bacterial isolates were tested for their resistance and sensitivity to 13 different antibiotics using disc diffusion method and the inhibition zone was measured according to Clinical Laboratory Standard Institutes guidelines [15]. 


\subsection{Detection of quorum sensing signal production}

Production of quorum sensing signal molecules (acyl homoserine lactones) was detected using gas chromatography mass spectrum (GC/MS) before and after treatment with Syzygium aromaticum extract. Acyl homoserine lactone was extracted from the bacterial culture as described in [16].

\subsection{Identification of the selected bacterial isolates by $16 \mathrm{~S}$ rRNA gene sequencing}

The most potent bacterial isolates were genetically identified by 16S rRNA gene sequencing at Sigma Scientific services Co., El Giza, Egypt.

\subsection{Data analysis}

Means and standard deviations of the studied variables were calculated. The differences among the means of the studied variables were tested using F-test. In addition, after testing the data for normality, one way and two way analysis of variance (ANOVA) was used to assess the significance of variations among the different variables using post hock according to SPSS software [17].

\section{Results and discussion}

\subsection{Preliminary Identification of clinical isolates}

Table (1): Biochemical tests for identification of the clinical isolates.

\begin{tabular}{|c|c|c|c|c|c|c|c|c|c|c|}
\hline 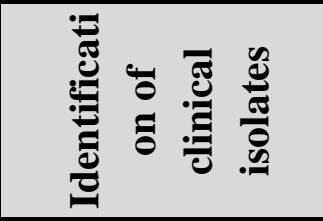 & & 串 & & 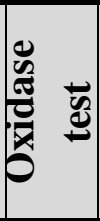 & 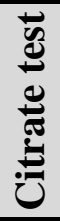 & 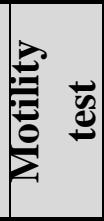 & $\begin{array}{l}\overrightarrow{\tilde{y}} \\
\stackrel{0}{0} \\
\frac{0}{0} \\
\stackrel{0}{\Xi}\end{array}$ & 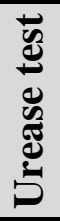 & 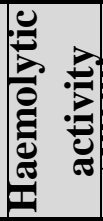 & 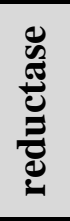 \\
\hline $\begin{array}{c}\text { Pseudomonas spp. } \\
\text { (44) }\end{array}$ & - & - & - & + & + & + & - & + & $\begin{array}{c}+ \\
\alpha / \beta\end{array}$ & + \\
\hline $\begin{array}{c}\text { Klebsiella spp. } \\
\text { (35) }\end{array}$ & + & + & + & - & + & - & V & + & $+\alpha$ & + \\
\hline $\begin{array}{l}\text { E. coli } \\
(18)\end{array}$ & + & + & + & - & - & + & + & - & V & + \\
\hline $\begin{array}{c}\text { Acinetobacter spp. } \\
\text { (17) }\end{array}$ & - & - & - & - & $\mathrm{V}$ & V & - & $\mathrm{V}$ & - & - \\
\hline $\begin{array}{c}\text { Proteus spp. } \\
\text { (8) }\end{array}$ & + & - & + & - & + & + & - & + & + & + \\
\hline $\begin{array}{c}\text { Enterobacter spp. } \\
\text { (3) }\end{array}$ & + & + & + & - & + & + & - & + & $+\alpha$ & + \\
\hline
\end{tabular}

+:positive result - : negative results Variable (V): some isolates produced positive results others produced negative ones. 


\subsection{Detection of biofilm formation}

Twenty seven isolates were positive biofilm forming in the three different methods of biofilm detection (Table 2), these isolates were tested for quorum sensing signal production.

Table (2): The percentage of positive biofilm forming isolates using different methods.

\begin{tabular}{|c|c|c|c|c|c|c|c|}
\hline \multirow{3}{*}{ Isolates } & \multicolumn{7}{|c|}{ Biofilm production } \\
\hline & \multicolumn{2}{|c|}{ CRA method } & \multirow{2}{*}{$\begin{array}{l}\text { Microtiter } \\
\text { plate assay } \\
\text { ( ELISA ) }\end{array}$} & \multicolumn{4}{|c|}{ Tube method } \\
\hline & $\begin{array}{l}\text { After } \\
24 \mathrm{~h}\end{array}$ & $\begin{array}{l}\text { After } \\
48 \mathrm{~h}\end{array}$ & & Weak & $\begin{array}{l}\text { Moderat } \\
\mathrm{e}\end{array}$ & $\begin{array}{l}\text { Stron } \\
\mathrm{g}\end{array}$ & $\begin{array}{l}\text { Total } \\
\text { no. }\end{array}$ \\
\hline Ps spp. & 12 & 12 & 11 & 0 & 1 & 5 & 6 \\
\hline$K$ spp. & 14 & 14 & 15 & 2 & 2 & 4 & 8 \\
\hline E. coli & 7 & 7 & 8 & 3 & 2 & 3 & 8 \\
\hline Ac spp. & 6 & 6 & 6 & 1 & 0 & 1 & 2 \\
\hline $\operatorname{Pr}$ spp. & 2 & 2 & 1 & 0 & 0 & 0 & 0 \\
\hline En spp. & 2 & 2 & 3 & 2 & 0 & 1 & 3 \\
\hline $\begin{array}{l}\text { Total } \\
\text { no. }\end{array}$ & 43 & 43 & 44 & 8 & 5 & 14 & 27 \\
\hline$\%$ & \multicolumn{2}{|l|}{34.4} & 35.2 & \multicolumn{4}{|l|}{21.6} \\
\hline
\end{tabular}

The previous results reported that using of more than one method was required for the test of biofilm formation; these results were correlated with [18, 19, $20 \& 21]$.
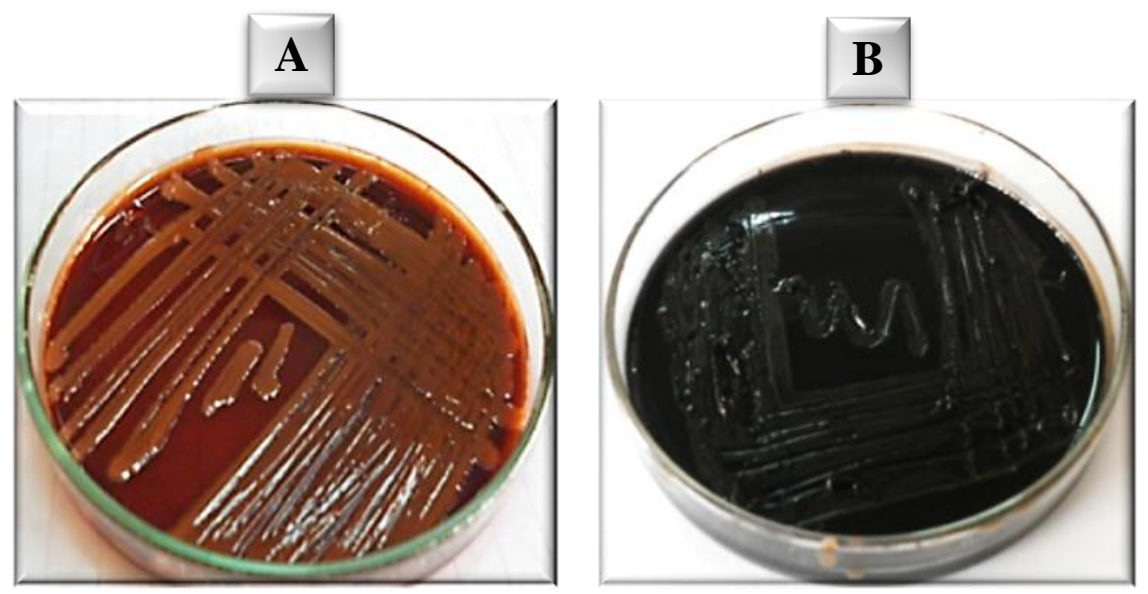

Figure (1): Biofilm formation test by different bacterial isolates using CRA medium. A: -ve biofilm formation (red color), B: +ve biofilm formation (black color). 


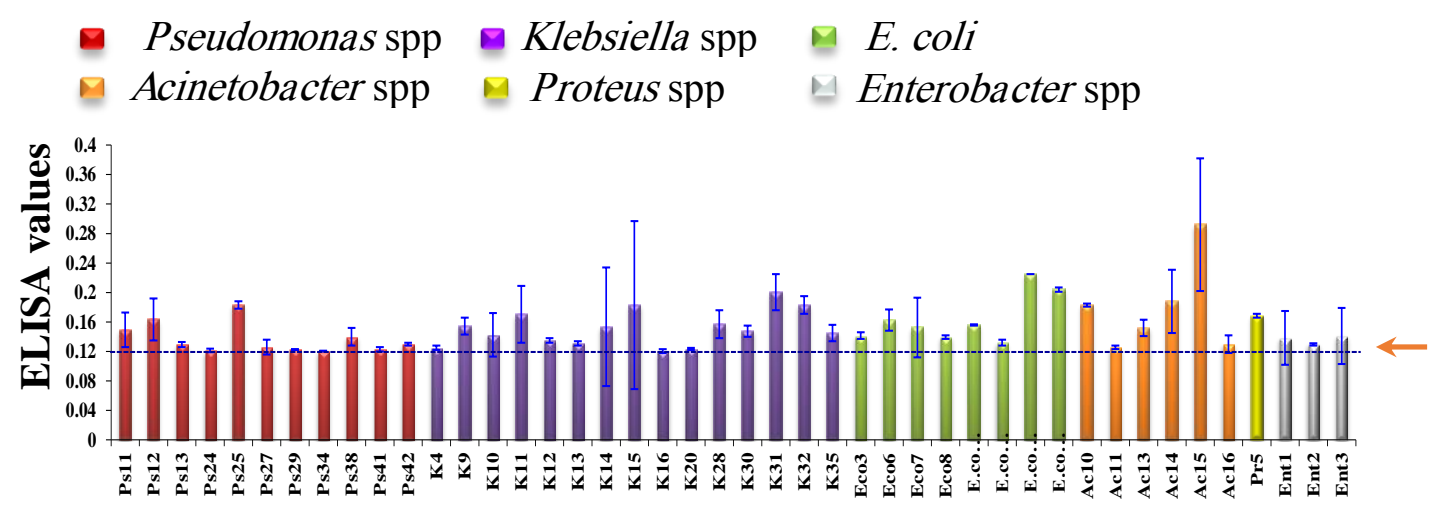

\section{Bacterial isolates}

Figure (2): Bacterial isolates showing positive biofilm formation by ELISA reader Ps: Pseudomonas; K: Klebsiella; E. coli: Escherichia coli; Ac: Acinetobacter; Pr: Proteus and En: Enterobacter. The arrow on the right side of the figure indicates the level of positive biofilm production (absorbance value $>0.12$ ).
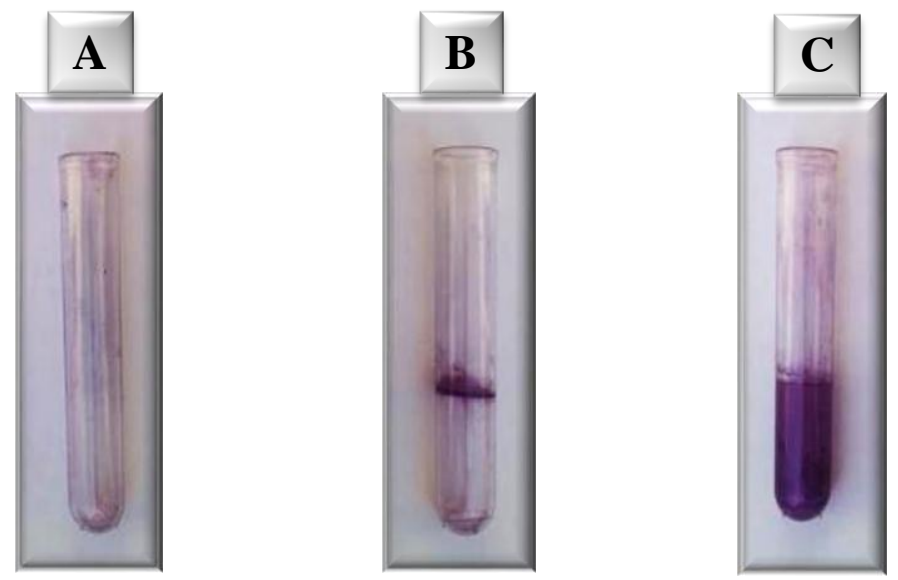

Figure (3): Positive and negative biofilm forming isolates using tube method.

$\mathrm{A}$ and $\mathrm{B}$ : -ve biofilm formation; $\mathrm{C}$ : +ve biofilm formation

\subsection{Antibiotic susceptibility test}

Sensitivity of the identified 20 clinical isolates towards different standard antibiotics was carried out to detect the multi-drug resistance of these isolates. Table (3) represented the susceptibility of the selected 20 clinical isolates to 13 different antibiotics. The sensitivity and resistance of the isolates were performed using the disk diffusion method. From the statistical analysis by $\mathrm{F}$ test, it showed that there is a highly significant difference between different 20 bacterial isolates with each other by the value $\mathrm{F}=2337 * * *, \mathrm{~F}=100798 * * *$ between different antibacterial agents (12 plant extracts and 13 antibiotics) with each other and $\mathrm{F}=773 * * *$ between different antibacterial agents (12 plant extracts and 13 antibiotics) and different 20 bacterial isolates at the probability $\mathrm{P}<0.001$. From the previous results, Pseudomonas sp41 
was the most resistant one against the all tested antibiotics followed by Pseudomonas sp42. For Klebsiella spp, the isolate $K 35$ was more resistant than $K 28$. For E. coli, the isolate $E$. coli13 was the most resistant one followed by E. coli16 and E. coli14. Finally the isolate Enterobacter sp3 was more resistant than En1 and En2 to the tested antibiotics.

The results obtained were confirmed by [22 \& 23], who found that erythromycin had the highest overall resistance and also most of the selected isolates (Pseudomonas spp., Klebsiella spp., E. coli and Enterobacter spp.) showed highly resistance against commonly used antibiotics.
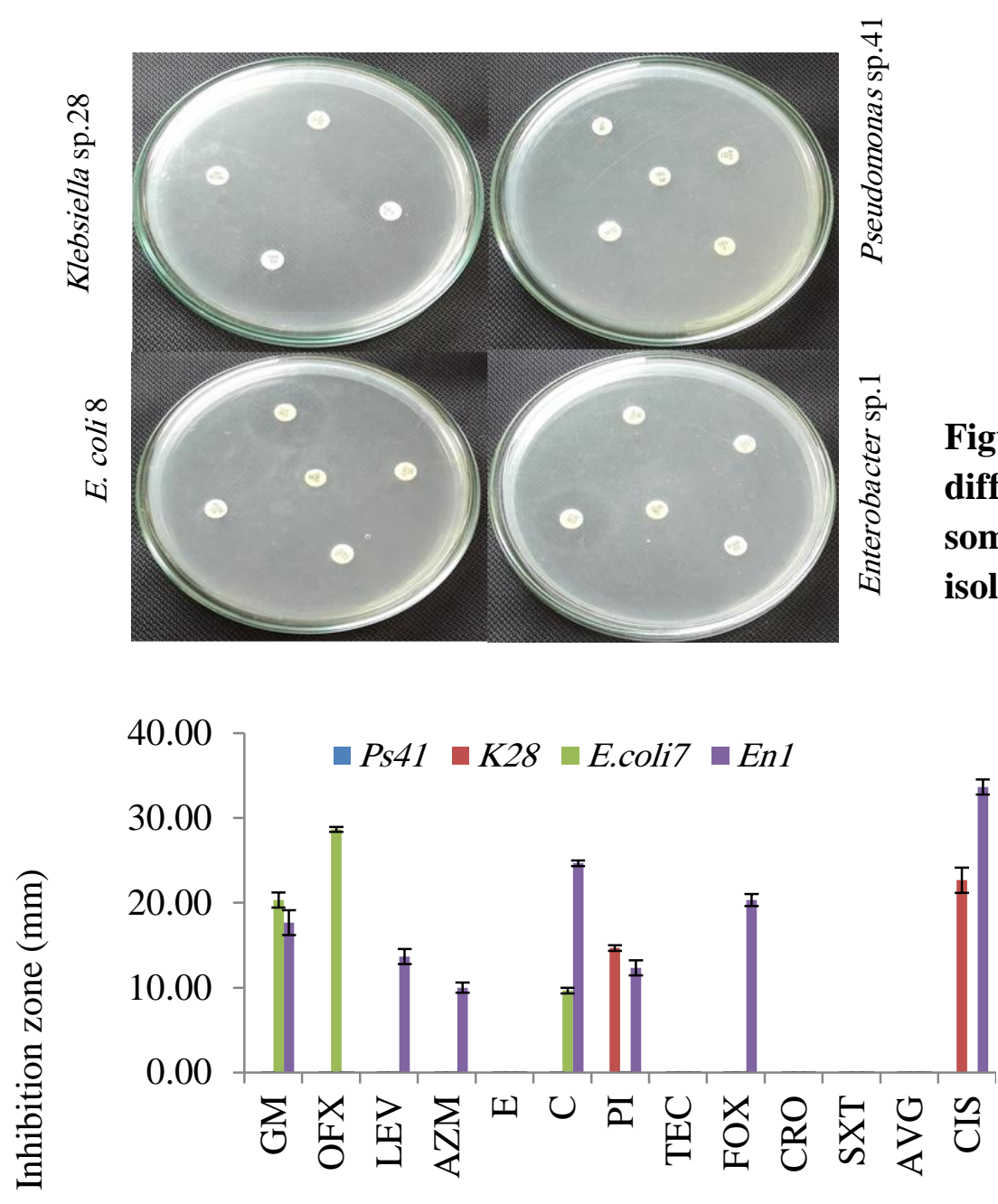

Figure (4): Effect of different antibiotics on some selected bacterial isolates.
Figure(5):

Antimicrobial activity of different antibiotics on Pseudomonas sp.41, Klebsiella sp.28, $E$. coli 7 and Enterobacter sp.1.

The antibiotics 
Table (3): Effect of different antibiotics on the biofilm forming bacterial isolates.

\begin{tabular}{|c|c|c|c|c|c|c|c|c|c|c|c|c|c|}
\hline \multirow[t]{2}{*}{ Isolate } & \multicolumn{13}{|c|}{ Inhibition zone (mm) } \\
\hline & GM & OFX & LEV & AZM & $\mathrm{E}$ & $\mathrm{C}$ & PI & TEC & FOX & $\mathrm{CRO}$ & SXT & AVG & CIS \\
\hline$P s 25$ & $15 \pm 0.6$ & $28 \pm 2.0$ & $30 \pm 1.5$ & 0 & 0 & 0 & $13 \pm 0.6$ & 0 & $15 \pm 0.6$ & $16 \pm 1.0$ & 0 & $12 \pm 0.6$ & $21 \pm 1.5$ \\
\hline$P s 41$ & 0 & 0 & 0 & 0 & 0 & 0 & 0 & 0 & 0 & 0 & 0 & 0 & 0 \\
\hline$P s 42$ & $11 \pm 0.6$ & $20 \pm 0.6$ & $39 \pm 1.7$ & 0 & 0 & $12 \pm 0.6$ & $16 \pm 1.0$ & 0 & 0 & 0 & $16 \pm 1.5$ & 0 & $12 \pm 1.5$ \\
\hline$K 9$ & 0 & 0 & $9 \pm 0.6$ & 0 & 0 & $16 \pm 0.6$ & $10 \pm 0.6$ & 0 & 0 & 0 & 0 & 0 & $11 \pm 1.5$ \\
\hline$K 12$ & $20 \pm 0.6$ & 0 & $10 \pm 0.6$ & 0 & 0 & $20 \pm 2.0$ & $11 \pm 0.6$ & 0 & 0 & 0 & 0 & 0 & $11 \pm 0.6$ \\
\hline$K 13$ & 0 & $26 \pm 0.6$ & 0 & 0 & 0 & $22 \pm 1.5$ & 0 & 0 & 0 & 0 & 0 & 0 & 0 \\
\hline$K 16$ & $18 \pm 0.6$ & 0 & $13 \pm 1.5$ & 0 & 0 & $20 \pm 0.6$ & $11 \pm 0.6$ & 0 & 0 & 0 & 0 & 0 & $24 \pm 1.5$ \\
\hline$K 28$ & 0 & 0 & 0 & 0 & 0 & 0 & $15 \pm 0.6$ & 0 & 0 & 0 & 0 & 0 & $23 \pm 2.5$ \\
\hline K35 & 0 & 0 & 0 & 0 & 0 & 0 & $14 \pm 1.2$ & 0 & 0 & 0 & 0 & 0 & $20 \pm 2.0$ \\
\hline E.coli3 & 0 & 0 & $10 \pm 1.2$ & $11 \pm 0.6$ & 0 & $20 \pm 3.5$ & 0 & 0 & $14 \pm 2.5$ & 0 & 0 & 0 & $12 \pm 0.6$ \\
\hline E.coli6 & $22 \pm 0.6$ & $30 \pm 1.5$ & $31 \pm 2.3$ & 0 & 0 & $18 \pm 2.5$ & $17 \pm 2.5$ & 0 & 0 & $13 \pm 1.5$ & $24 \pm 1.5$ & 0 & $28 \pm 1.5$ \\
\hline E.coli7 & $20 \pm 1.5$ & $29 \pm 0.6$ & 0 & 0 & 0 & $10 \pm 0.6$ & 0 & 0 & 0 & 0 & 0 & 0 & 0 \\
\hline E.coli8 & $21 \pm 1.2$ & $30 \pm 2.5$ & $29 \pm 1.2$ & 0 & 0 & $17 \pm 1.5$ & $16 \pm 1.5$ & 0 & 0 & $10 \pm 0.6$ & $24 \pm 0.6$ & 0 & $24 \pm 0.6$ \\
\hline E.coli13 & $14 \pm 0.6$ & 0 & 0 & 0 & 0 & $20 \pm 0.6$ & 0 & 0 & 0 & 0 & 0 & 0 & 0 \\
\hline E.coli14 & $18 \pm 1.0$ & 0 & 0 & 0 & 0 & $19 \pm 1.2$ & 0 & 0 & 0 & 0 & 0 & 0 & 0 \\
\hline E.coli15 & $15 \pm 0.6$ & 0 & 0 & 0 & 0 & $15 \pm 3.1$ & 0 & 0 & $13 \pm 0.6$ & 0 & $14 \pm 1.5$ & 0 & $13 \pm 1.2$ \\
\hline E.coli16 & $16 \pm 2.5$ & 0 & 0 & 0 & 0 & $20 \pm 2.5$ & 0 & 0 & 0 & 0 & 0 & 0 & 0 \\
\hline$E n 1$ & $18 \pm 2.5$ & 0 & $14 \pm 1.5$ & $10 \pm 1.0$ & 0 & $25 \pm 0.6$ & $12 \pm 1.5$ & 0 & $20 \pm 1.2$ & 0 & 0 & 0 & $34 \pm 1.5$ \\
\hline En2 & $16 \pm 0.6$ & 0 & $11 \pm 0.6$ & 0 & 0 & $20 \pm 2.5$ & $9 \pm 0.6$ & 0 & $12 \pm 0.6$ & 0 & 0 & $10 \pm 1.5$ & $16 \pm 0.6$ \\
\hline En3 & $19 \pm 1.2$ & 0 & 0 & $10 \pm 0.6$ & 0 & $21 \pm 1.2$ & 0 & 0 & $16 \pm 0.6$ & 0 & 0 & 0 & $13 \pm 1.0$ \\
\hline
\end{tabular}




\subsection{Plant extracts and inhibition of biofilm formation}

\subsubsection{Antibacterial activity of some plant extracts}

Ethyl acetate extracts of 12 plant species were tested against the 20 bacterial isolates that showed both biofilm and QS signal production, using the standard well agar diffusion method.

Each bacterial isolate was inhibited by certain plant extracts, then the most effective plant extracts were tested for their anti-biofilm activity on each bacterial isolate using tube method. Data in table 9 showed that, Syzygium aromaticum extract was the most effective one against almost of the tested selected bacterial isolates, followed by Allium sativum, but Ocimum sp. extract showed highly activity against isolates $P s 41, K 9$ and E. coli6 than Syzygium aromaticum one. Also Illicium verum was more active against the isolate Ps41 than Syzygium aromaticum.

The results of the present study were in harmony with the study carried out by [24 \& $25]$ in which Clove extract exhibited antibacterial activity against all tested bacterial isolates.

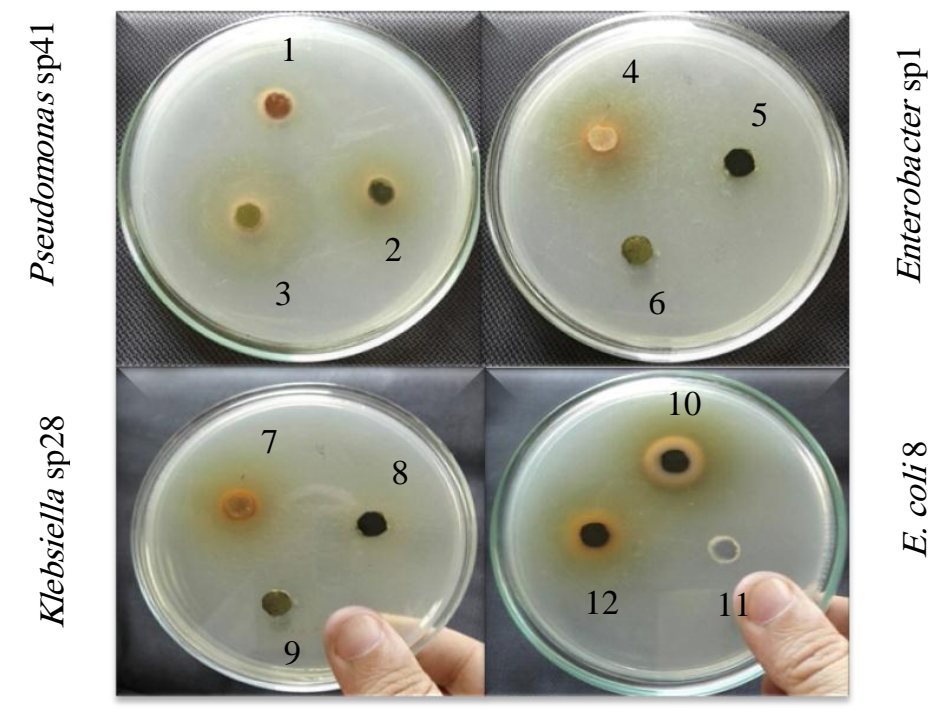

Figure (6): Effect of plant extracts on the selected bacterial isolates.

1: Illicium sp.; 2: Callistemon sp.; 3: Ocimum sp.; 4: Syzygium sp.; 5: Moringa sp.; 6: Clerodendrum sp.; 7: Syzygium aromaticum; 8: Moringa sp.; 9: Clerodendrum sp.; 10: Callistemon sp.; 11: DMSO and 12: Melaleuca sp 


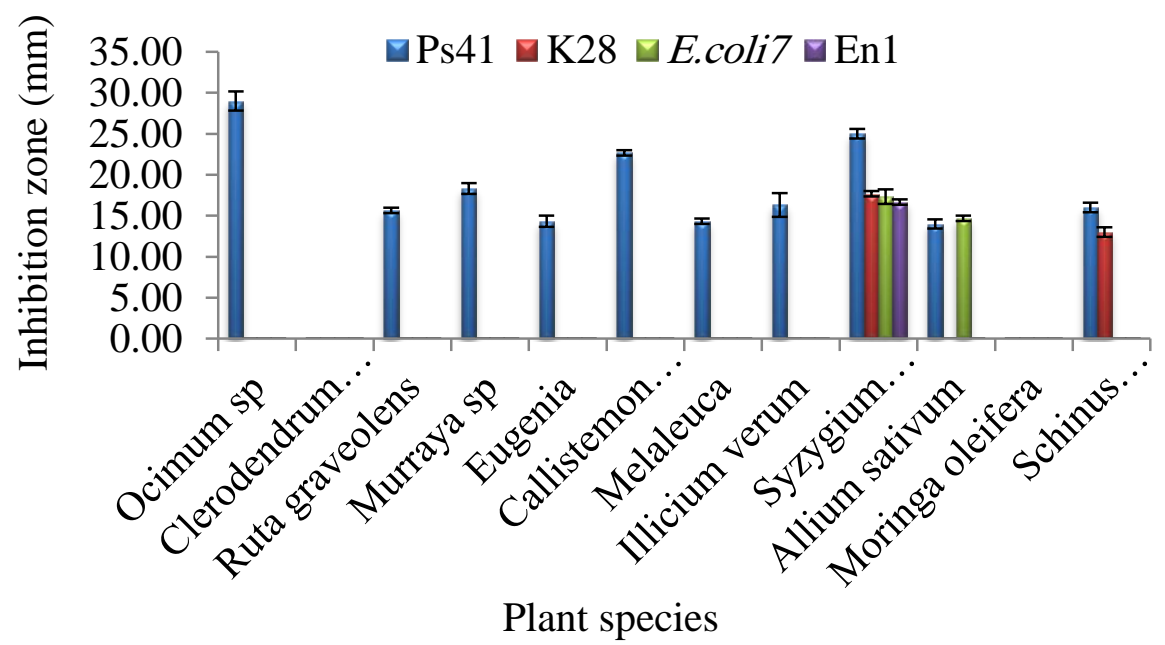

Figure (7):

Antimicrobia activity of different ethyl acetate plant extracts on Pseudomonas sp.41, Klebsiella sp.28, $E$. coli 7 and Enterobacter sp.1.

\subsubsection{Plant extracts and inhibition of biofilm formation}

The most effective two plant extracts against each bacterial isolate as antibacterial agent were selected and tested for their antibiofilm activity against this biofilm forming and quorum sensing producing bacterial isolate using tube method. This selection was according to the previous results obtained in Table (4). Five plant extracts were selected for this test as shown in Table (5).

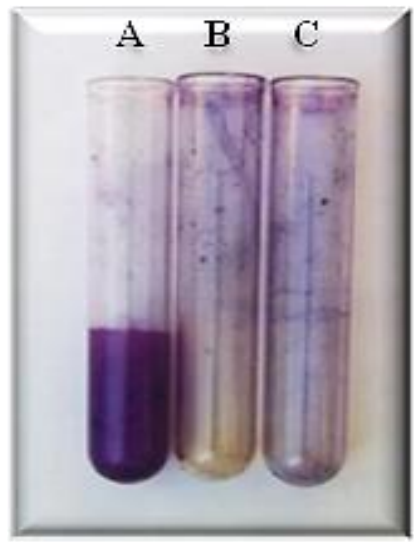

Figure (8): Antibiofilm activity of Dianthus sp. and Allium sativum extracts on Pseudomonas sp. isolate no. 25 A: +ve biofilm (before treatment), B: -ve biofilm after treatment with Syzygium aromaticum extract, C: -ve biofilm after treatment with Allium sativum extract. 
Table (4): The antibacterial activity of each ethyl acetate plant extract against twenty clinical isolates

\begin{tabular}{|c|c|c|c|c|c|c|c|c|c|c|c|c|}
\hline \multirow[b]{2}{*}{ Isolate } & \multicolumn{12}{|c|}{ Inhibition zones (mm) } \\
\hline & $\begin{array}{l}0 \\
5 \\
5 \\
0 \\
0 \\
0\end{array}$ & 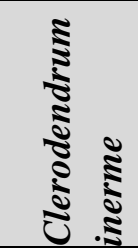 & 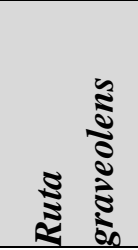 & 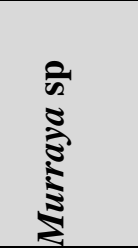 & 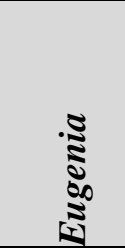 & 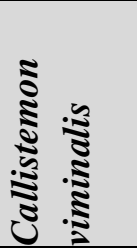 & $\frac{\sqrt{4}}{3}$ & 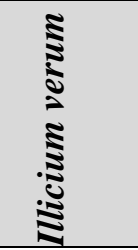 & 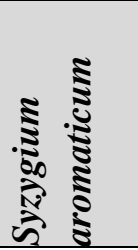 & 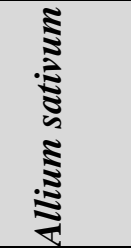 & 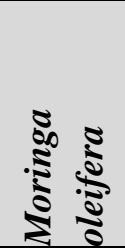 & 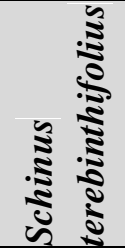 \\
\hline$P s 25$ & 0 & 0 & 0 & 0 & 0 & 0 & 0 & $14 \pm 1.0$ & $18 \pm 0.6$ & $21 \pm 0.6$ & 0 & 0 \\
\hline$P s 41$ & $29 \pm 2.0$ & 0 & $16 \pm 0.6$ & $18 \pm 1.2$ & $14 \pm 1.2$ & $23 \pm 0.6$ & $14 \pm 0.6$ & $16 \pm 2.5$ & $25 \pm 1.0$ & $14 \pm 1.0$ & 0 & $16 \pm 1.0$ \\
\hline$P s 42$ & $14 \pm 0.0$ & $11 \pm 1.0$ & $11 \pm 1.2$ & $12 \pm 0.6$ & $12 \pm 0.6$ & $13 \pm 1.0$ & $12 \pm 1.0$ & $18 \pm 0.6$ & $15 \pm 1.5$ & $12 \pm 1.0$ & 0 & $13 \pm 1.5$ \\
\hline$K 9$ & $22 \pm 2.0$ & 0 & $12 \pm 0.6$ & $12 \pm 0.6$ & $12 \pm 1.0$ & $15 \pm 2.5$ & $11 \pm 1.2$ & $15 \pm 1.0$ & $16 \pm 0.6$ & $12 \pm 2.0$ & 0 & $14 \pm 1.5$ \\
\hline$K 12$ & $13 \pm 0.6$ & $12 \pm 0.6$ & 0 & 0 & 0 & $14 \pm 1.5$ & $12 \pm 2.5$ & $14 \pm 0.6$ & $17 \pm 1.0$ & $15 \pm 1.5$ & 0 & $12 \pm 1.0$ \\
\hline$K 13$ & $14 \pm 1.0$ & 0 & 0 & 0 & $13 \pm 1.5$ & $14 \pm 1.0$ & $13 \pm 1.5$ & $14 \pm 1.5$ & $16 \pm 2.5$ & $15 \pm 0.6$ & 0 & $13 \pm 0.6$ \\
\hline$K 16$ & 0 & 0 & 0 & 0 & 0 & $12 \pm 1.5$ & $12 \pm 0.6$ & 0 & $18 \pm 1.5$ & $19 \pm 1.5$ & 0 & $14 \pm 1.5$ \\
\hline$K 28$ & 0 & 0 & 0 & 0 & 0 & 0 & 0 & 0 & $18 \pm 0.6$ & 0 & 0 & $13 \pm 1.0$ \\
\hline$K 35$ & 0 & 0 & 0 & 0 & $12 \pm 0.6$ & $12 \pm 1.2$ & $12 \pm 1.0$ & 0 & $16 \pm 1.0$ & $20 \pm 1.0$ & 0 & $14 \pm 1.0$ \\
\hline E.coli6 & $22 \pm 1.2$ & 0 & $15 \pm 0.6$ & $13 \pm 1.0$ & 0 & $14 \pm 0.6$ & 0 & $15 \pm 0.6$ & $17 \pm 0.6$ & $14 \pm 1.5$ & $14 \pm 0.6$ & 0 \\
\hline E.coli7 & 0 & 0 & 0 & 0 & 0 & 0 & 0 & 0 & $17 \pm 1.5$ & $15 \pm 0.6$ & 0 & 0 \\
\hline E.coli8 & $23 \pm 0.6$ & 0 & 0 & 0 & $18 \pm 1.5$ & $22 \pm 0.6$ & $18 \pm 0.6$ & 0 & $34 \pm 1.5$ & $25 \pm 1.5$ & $14 \pm 1.5$ & $20 \pm 2.5$ \\
\hline E.coli13 & 0 & 0 & 0 & 0 & 0 & $15 \pm 0.6$ & $15 \pm 0.6$ & 0 & $17 \pm 0.6$ & $18 \pm 0.6$ & 0 & $15 \pm 1.0$ \\
\hline E.coli14 & 0 & 0 & 0 & 0 & 0 & $14 \pm 1.5$ & $12 \pm 1.0$ & 0 & $16 \pm 2.0$ & $18 \pm 1.2$ & 0 & $13 \pm 0.6$ \\
\hline E.coli15 & $15 \pm 1.0$ & 0 & 0 & $16 \pm 0.6$ & $15 \pm 1.2$ & $15 \pm 0.6$ & $15 \pm 0.6$ & $13 \pm 0.6$ & $20 \pm 1.5$ & $26 \pm 0.6$ & 0 & $15 \pm 1.5$ \\
\hline E.coli16 & $12 \pm 2.5$ & 0 & 0 & $13 \pm 1.5$ & $12 \pm 1.5$ & $12 \pm 1.0$ & $12 \pm 1.0$ & 0 & $17 \pm 0.6$ & $18 \pm 0.6$ & 0 & $12 \pm 1.5$ \\
\hline En1 & 0 & 0 & 0 & 0 & 0 & 0 & 0 & 0 & $17 \pm 0.6$ & 0 & 0 & 0 \\
\hline$E n 2$ & $14 \pm 1.5$ & 0 & 0 & 0 & 0 & $14 \pm 1.5$ & $12 \pm 2.0$ & $13 \pm 1.0$ & $18 \pm 0.6$ & $15 \pm 1.0$ & 0 & $13 \pm 0.6$ \\
\hline En3 & 0 & 0 & 0 & 0 & 0 & $11 \pm 1.2$ & $12 \pm 0.6$ & $12 \pm 1.0$ & $17 \pm 1.0$ & $19 \pm 1.5$ & 0 & $14 \pm 1.5$ \\
\hline
\end{tabular}

Ps: Pseudomonas; K: Klebsiella; E. coli: Escherichia coli and En: Enterobacter 
J. Sci. Res. Sci.,Vol.(35), 2018

Table (5): Activity of plant extracts on biofilm production in each bacterial isolate.

\begin{tabular}{|c|c|c|c|c|c|c|c|c|c|c|c|c|c|c|c|c|c|c|c|c|c|c|c|c|c|}
\hline \multirow{3}{*}{ MO } & \multicolumn{25}{|c|}{ Plant extract and biofilm production } \\
\hline & \multicolumn{5}{|c|}{ Ocimum sp } & \multicolumn{5}{|c|}{ Illicium verum } & \multicolumn{5}{|c|}{ Syzygium aromaticum } & \multicolumn{5}{|c|}{ Allium sativum } & \multicolumn{5}{|c|}{ Schinus terebinthifolius } \\
\hline & $\begin{array}{l}0.0 \\
5 \\
\mathrm{ml}\end{array}$ & $\begin{array}{l}0 . \\
1 \\
\mathrm{ml}\end{array}$ & $\begin{array}{l}0 . \\
2 \\
\mathrm{ml}\end{array}$ & $\begin{array}{l}0 . \\
5 \\
\mathrm{ml}\end{array}$ & $\begin{array}{l}1 \\
\mathrm{ml}\end{array}$ & $\begin{array}{l}0.0 \\
5 \\
\mathrm{ml}\end{array}$ & $\begin{array}{l}0 . \\
1 \\
\mathrm{ml}\end{array}$ & $\begin{array}{l}0 . \\
2 \\
\mathrm{ml}\end{array}$ & $\begin{array}{l}0 . \\
5 \\
\mathrm{ml}\end{array}$ & $\begin{array}{l}1 \\
\mathrm{ml}\end{array}$ & $\begin{array}{l}0.0 \\
5 \\
\mathrm{ml}\end{array}$ & $\begin{array}{l}0 . \\
1 \\
\mathrm{ml}\end{array}$ & $\begin{array}{l}0 . \\
2 \\
\mathrm{ml}\end{array}$ & $\begin{array}{l}0 . \\
5 \\
\mathrm{ml}\end{array}$ & $\begin{array}{l}1 \\
\mathrm{ml}\end{array}$ & $\begin{array}{l}0.0 \\
5 \\
\mathrm{ml}\end{array}$ & $\begin{array}{l}0 . \\
1 \\
\mathrm{ml}\end{array}$ & $\begin{array}{l}0 . \\
2 \\
\mathrm{ml}\end{array}$ & $\begin{array}{l}0 . \\
5 \\
\mathrm{ml}\end{array}$ & $\begin{array}{l}1 \\
\mathrm{ml}\end{array}$ & $\begin{array}{l}0.0 \\
5 \\
\mathrm{ml}\end{array}$ & $\begin{array}{l}0 . \\
1 \\
\mathrm{ml}\end{array}$ & $\begin{array}{l}0 . \\
2 \\
\mathrm{ml}\end{array}$ & $\begin{array}{l}0 . \\
5 \\
\mathrm{ml}\end{array}$ & $\begin{array}{l}1 \\
\mathrm{ml}\end{array}$ \\
\hline Ps 25 & & & & & & & & & & & + & - & - & - & - & + & - & - & - & - & & & & & \\
\hline$P s 41$ & + & + & + & - & - & & & & & & + & - & - & - & - & & & & & & & & & & \\
\hline Ps42 & & & & & & + & - & - & - & - & + & - & - & - & - & & & & & & & & & & \\
\hline$K 9$ & + & + & + & - & - & & & & & & + & - & - & - & - & & & & & & & & & & \\
\hline$K 12$ & & & & & & & & & & & - & - & - & - & - & + & + & - & - & - & & & & & \\
\hline$K 13$ & & & & & & & & & & & + & - & - & - & - & + & + & - & - & - & & & & & \\
\hline$K 16$ & & & & & & & & & & & + & - & - & - & - & + & - & - & - & - & & & & & \\
\hline$K 28$ & & & & & & & & & & & - & - & - & - & - & & & & & & + & - & - & - & - \\
\hline$K 35$ & & & & & & & & & & & - & - & - & - & - & + & + & - & - & - & & & & & \\
\hline$E 3$ & & & & & & & & & & & + & - & - & - & - & + & + & - & - & - & & & & & \\
\hline$E 6$ & + & + & - & - & - & & & & & & - & - & - & - & - & & & & 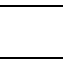 & & & & & & \\
\hline$E 7$ & & & & & & & & & & & - & - & - & - & - & + & - & - & - & - & & & & & \\
\hline$E 8$ & & & & & & & & & & & - & - & - & - & - & + & - & - & - & - & & & & & \\
\hline$E 13$ & & & & & & & & & & & + & - & - & - & - & + & + & - & - & - & & & & & \\
\hline$E 14$ & & & & & & & & & & & - & - & - & - & - & + & - & - & - & - & & & & & \\
\hline$E 15$ & & & & & & & & & & & + & - & - & - & - & + & - & - & - & - & & & & & \\
\hline$E 16$ & & & & & & & & & & & - & - & - & - & - & + & - & - & - & - & & & & & \\
\hline$E n 1$ & & & & & & & & & & & - & - & - & - & - & & & & & & & & & & \\
\hline En2 & & & & & & & & & & & + & - & - & - & - & + & - & - & - & - & & & & & \\
\hline En3 & & & & & & & & & & & - & - & - & - & - & + & + & - & - & - & & & & & \\
\hline
\end{tabular}

MO: microorganism; Ps: Pseudomonas; K: Klebsiella; E: Escherichia coli and En: Enterobacter 
Syzygium aromaticum extract was the most effective one as a biofilm inhibitor by the concentration ranged between $0.05-0.1 \mathrm{ml}$ against the all twenty selected bacterial strains, followed by Allium sativum which was effective by the concentration ranged between 0.1-0.2 $\mathrm{ml}$. Then Syzygium aromaticum extract was tested for inhibition of quorum sensing signal (AHLs) production in the selected twenty bacterial strains.

\subsection{Quorum sensing signal production before and after treatment with Clove extract}

Quorum sensing signal (AHLs) production was inactivated by Syzygium aromaticum extract in the selected bacterial strains, and so no lactone signal was detected using GC/MS. The concentration of Syzygium aromaticum extract was $20 \mu \mathrm{l} / \mathrm{ml}$.

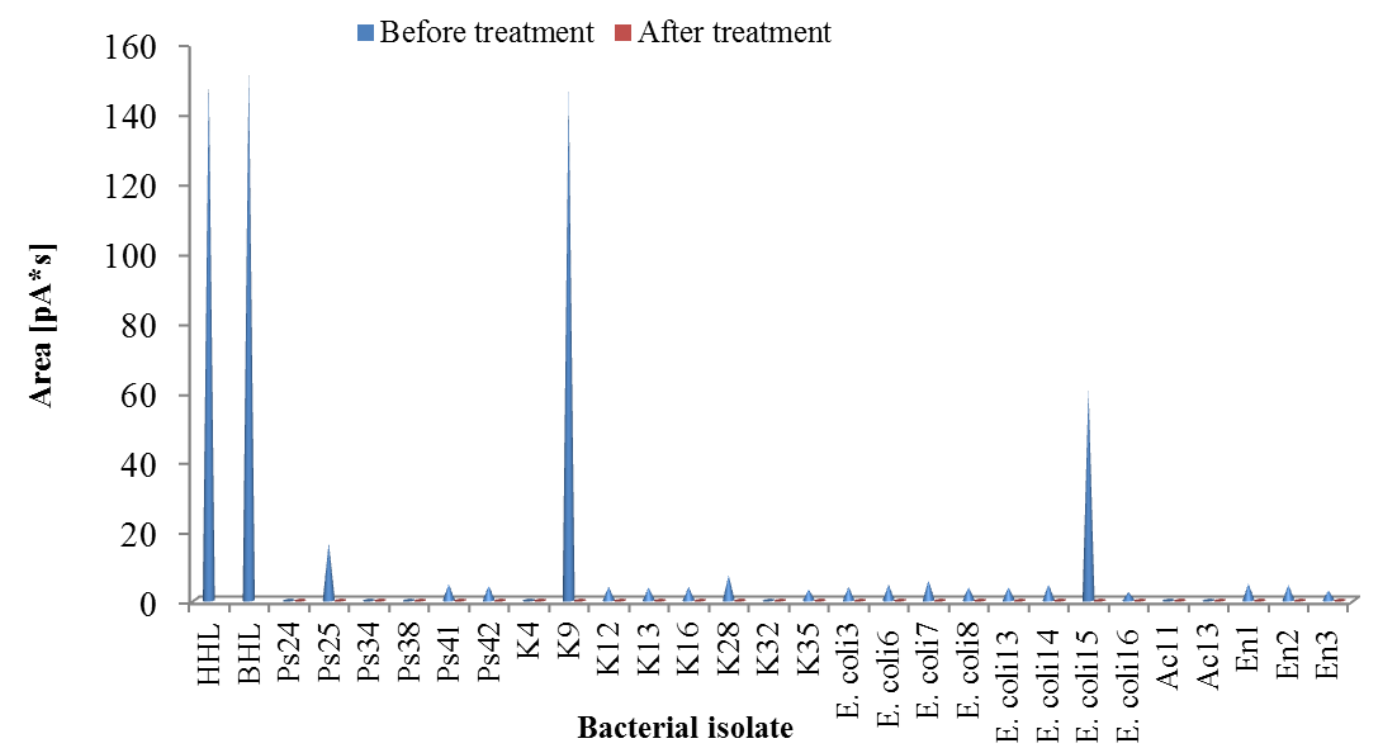

Figure (9): GC/MS of Q.S signal (AHLs) production before and after treatment with Syzygium aromaticum extract.

\subsection{Molecular analysis}

The most virulent bacterial isolates were genetically identified by $16 \mathrm{~S}$ rRNA gene sequencing. The identified strains were presented in (Table 6).

Table (6): 16S rRNA gene sequencing of 4 selected bacterial isolates

\begin{tabular}{|l|l|l|}
\hline Isolate No. & Blast identity & NCBI accession No. \\
\hline FS7 & Escherichia coli FS7 & MF197876 \\
\hline FPF28 & Klebsiella pneumonia FPF28 & MF197877 \\
\hline FS1 & Enterobacter cloacae FS1 & MF197878 \\
\hline FS41 & Pseudomonas aeruginosa FS41 & MF197879 \\
\hline
\end{tabular}




\section{Conclusions}

The results reported that using of some medicinal plants is safer and more effective than using agents of chemical origin to inhibit quorum sensing and biofilm formation in the studied pathogenic Gram negative bacteria. These bacteria not only are the common cause of nosocomial infection but also are considered to be multi-drug resistant bacteria as a result of formation of biofilm. Clove extract showed highly antibacterial activity and inhibit both biofilm formation and quorum sensing system in the studied bacterial isolates.

\section{References}

Fox LK, Zadoks RN, Gaskins CT (2005). Biofilm production by Staphylococcus aureus associated with intramammary infection. Vet Microbiol, 107: 295-99.

Vancraeynest, D., Hermans, K. and Haesenbrouck, F. (2004). Genotypic and phenotypic screening of high and low virulence Staphylococcus aureus isolatesfrom rabbit for biofilm formation and MSCRAMMs. Vet Microbiol, 103: 241-47.

Thomas, D and Day, F. (2007). Biofilm formation by plant associated bacteria. Ann Rev Microbiol; 61:401-22.

Research on microbial biofilms (PA-03-047) (2002). NIH, National Heart, Lung, and Blood Institute. -12-20.

Fuqua, W.C.; Greenberg, E.P. (2002) Listening in on bacteria: Acyl-homoserine lactone signaling. Nature Rev. Mol. Cell Biol., 3, 685-695.

Waters, C.M.; Bassler, B.L. (2005) Quorum sensing: Cell-to-cell communication in bacteria. Annu. Rev. Cell. Dev. Biol., 21, 319-346.

Camilli, A.; Bassler, B.L. (2006) Bacterial small-molecule signaling pathways. Science, 311, 1113-1116.

Williams, P. (2007). Quorum sensing, communication and cross-kingdom signalling in the bacterial world. Microbiology: 153, 3923-3938.

Hanzelka, B.L. (1995) Greenberg, E.P. Evidence that the N-terminal region of the Vibrio fischeri LuxR protein constitutes an autoinducer-binding domain. J. Bacteriol., 177, 815-817.

Krieg, N.R. and Holt, J.G. (1994). In: Bergey's Manual of Determinative Bacteriology. Baltimore, Williams and Wilkins Co., Library of Congress

Freeman J, Falkiner FR, Keane CT (1989). New method for detecting

slime production by coagulase negative staphylococci. J Clin Pathol; 42:872-4.

Cramton SE, Gerke C, Gotz F (2001). In vitro method to study Staphylococcal biofilm formation. Methods Enzymol, 336: 239-55. 
Christensen GD, Simpson WA, Bisno AL, Beachey EH (1982). Adherence of slime producing strains of Staphylococcus epidermidis to smooth surfaces. Infect Immun; 37:31826.

Hewitt, W. and Vincent, S. (2003). Theory and Application of Microbiological Assay. Academic Press (Inc), London, UK, ISBN0849318246.

Clinical and Laboratory Standards Institute (CLSI) (2017). Performance Standards for Antimicrobial Disc Susceptibility Tests. CLSI document M100-S17 (ISBNI-56238-625-5). Pennsylvania, USA.

Shaw, P.D.; Ping, G.; Daly, S.L.; Cha, C.; Cronan, J.E., Jr.; Rinehart, K.L (1997). Detecting and characterizing $\mathrm{N}$-acyl-homoserina lactone signal molecules by thin layer chromatography. Proc. Nathl. Acad. Sci. USA., 94, 6036-6041.

SPSS, 2006. SPSS base 15.0User's guide. SPSS inc., Chicago, USA.

Christensen GD, Simpson WA, Bisno AL, Beachey EH. (1982). Adherence of slime producing strains of Staphylococcus epidermidis to smooth surfaces. Infect Immun; 37:31826.

\section{Mathur T, Singhal S, Khan S, Upadhyay DJ, Fatma T, Rattan A. (2006).}

Detection of biofilm formation among the clinical isolates of staphylococci: an evaluation of three different screening methods. Indian J Med Microbiol; 24(1) : 25-9.

Bose S, Khodke M, Basak S, Mallick SK. (2009). Detection of biofilm producing staphylococci: need of the hour. J Clin Diagn Res; 3:1915-20.

Ruzicka F, Hola V, Votava M et al., (2004). Biofilm detection and clinical significance of Staphylococcus epidermidis isolates. Folia Microbiol (Praha); 49(5):596-600.

Ebenebe, J. C,; Emeka-Okafor, K. M.; Akujobi, C. N.; Ezeanya, C. C. and Agbakoba, N. R. (2014). Egwuatu Plasmid Profile of Uropathogens among Children British Journal and Medical Research 4(5): 1195-1203.

Kibret, M. and Abera, B. (2014). Prevalence and antibiogram of bacterial isolates from urinary tract infections at Dessie Health Research Laboratory, Ethiopia Asian Pac. J. Trop Biomed; 4(2): 164-168.

Muafia, S.; Khan, S. J.; Zahid, N. and Khan, N. H. (2010). Appraisal of Nutritional status and Antimicrobial Activity of Clove, Kalonji, Cinnamon, Black Pepper and Sweet WEET Basil ASI Pharmacologyonline, 2: 591-599.

Ram, K. P.; Pranay, J. and Chetan, Sh. (2010). Antimicrobial Activity of Ethanolic Extracts of Syzygium aromaticum and Allium sativum Against Food Associated Bacteria and Fungi. Ethanobotanical Leaflets, 14: 344-60. 


\section{الملخص باللغة العربية}

تثبيط تكوين الغشاء الحيوى فى بعض البكتريا الممرضه السالبه لصبغة جرام باستخدام مركبات مضاده للحس العددى

فاطمه عبد التواب عبد العليم شعبان ، زينب محمد حسن خيرالله ، عبير احمد رشدى ،

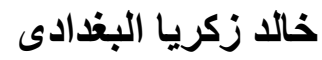

$$
\text { قسم النبات ـ كلية البنات للآداب و العلوم والتربيه - جامعة عين شمس }
$$

فى هذه الدر اسة تم تجميع هب ا عزله بكيترية من معمل الميكروبيولوجى بمستشفى الدمرداش بمحافظة القاهرة. - اهـ وقد تم عزل هذه البكتريا من مصادر مختلفه مثل الصديد ، اللعاب ، افر ازات الانف ، الدم والبول. تبعاً لصفات الثكل و النمو على الوسط والتشخيص الكيمو حيوي تم تعريفهم إلى ؟ ؟ عزله له بنتمون

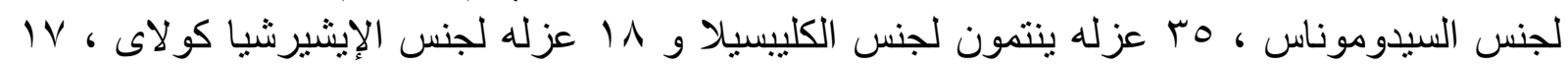

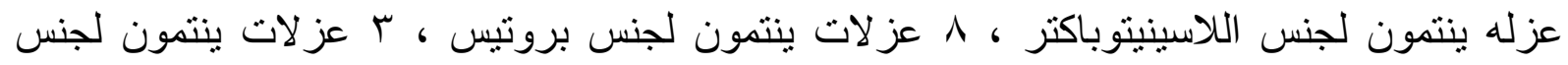
الانتيروباكتر.

وتم تعبين قدرة هذه العزلات البكتيريه على تكوين الغثاء الحيوى بثلاث طرق مختلفه وكانت

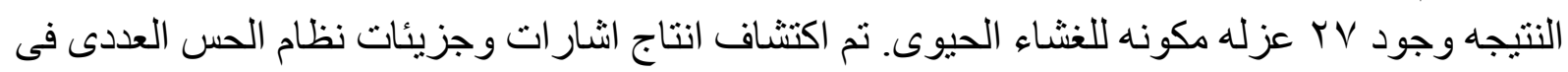

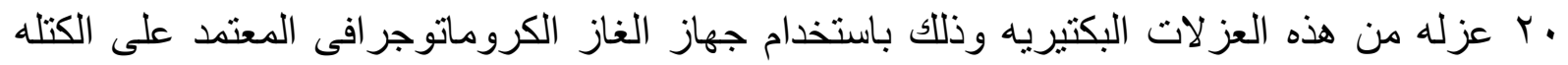

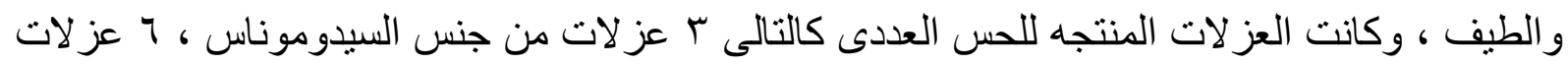
من جنس الكليبيلا ، ^ عز لات من جنس الإيشيرشيا كولاى و ب عز لاتلات من جنس الانتيروباكتر.

تم اجراء اختبار النشاط المضاد لتكوين الغشاء الحيوى لبعض المستخلصات النباتيه باستخدام طريقة الانابيب حيث أظهرت النتائج ان مستخلص نبات القرنفل هو الاكثر فاعليه فى تثبيط تكوين الغثاء

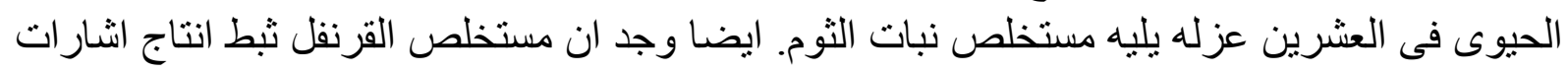
وجزيئات نظام الحس العددى فى كل من العشرين عزله له له

من النتائج الموضحه اعلاه، تم ترشيح مستخلص نبات القرنفل لاستخدامه كماده فعاله و عامل

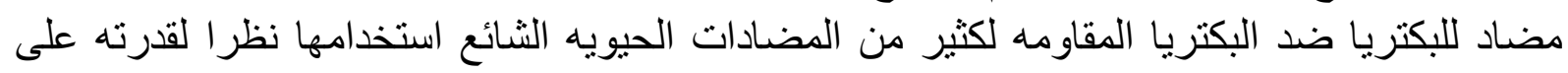
تثبيط تكوين الغشاء الحيوى وانتاج اشارات وجزيئات نظام الحس العددى ــ وفى النهايه تم تعريف

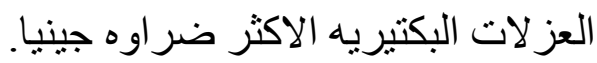

